INPLASY

PROTOCOL

To cite: Wang et al. Relevance of dynamic cerebral autoregulation after acute ischemic stroke with prognosis: A protocol for systematic review and metaanalysis. Inplasy protocol 202210056. doi:

10.37766/inplasy2022.1.0056

Received: 11 January 2022

Published: 11 January 2022

Corresponding author:

Kai Wang

550624487@qq.com

Author Affiliation:

Changchun University of

Chinese Medicine.

Support: No. 2019YFC1709902.

Review Stage at time of this submission: The review has not yet started.

Conflicts of interest:

None declared.

\section{Relevance of dynamic cerebral autoregulation after acute ischemic stroke with prognosis: A protocol for systematic review and meta-analysis}

Wang, K¹ Lv, Z2; Xu, P3; Wang, B4; Hou, A5; Lan, T6; Zhang, D7; Wang, J8.

Review question / Objective: Is there a relevance between dCA and AIS prognosis? The effect of the extent of damage in dCA on AIS prognosis?

Condition being studied: Relevance of dynamic cerebral autoregulation after acute ischemic stroke with prognosis.

Eligibility criteria: Eligible studies must meet the following criteria: (1) Longitudinal or cross-sectional observational studies, with a TFA assessment and prognosis of acute ischemic stroke; (2) Have clear diagnostic criteria; (3) TFA that includes a measure of gain, phase, coherence; (4) The first dCA assessment for ischemic stroke to be done within 24 hours of onset by TFA; (5) Clear reports of prognosis expressed as Modified Rankin Scale(mRS).

INPLASY registration number: This protocol was registered with the International Platform of Registered Systematic Review and Meta-Analysis Protocols (INPLASY) on 11 January 2022 and was last updated on 11 January 2022 (registration number INPLASY202210056).

\section{INTRODUCTION}

Review question / Objective: Is there a relevance between dCA and AIS prognosis? The effect of the extent of damage in dCA on AIS prognosis?
Condition being studied: Relevance of dynamic cerebral autoregulation after acute ischemic stroke with prognosis.

\section{METHODS}

Participant or population: All people who have ischemic stroke defined by American 
Heart Association / American Stroke Association (AHA/ASA) criteria.

Intervention: TFA includes a measure of gain, phase, coherence. We will also include studies that have examined any of the above, but not all three measures. We will only include TFA in the context of cerebral autoregulation with ischemic stroke patients and not in other diseases.

Comparator: Healthy individuals with dCA were assessed using TFA or no controls.

Study designs to be included: Observational study.

Eligibility criteria: Eligible studies must meet the following criteria: (1) Longitudinal or cross-sectional observational studies, with a TFA assessment and prognosis of acute ischemic stroke; (2) Have clear diagnostic criteria; (3) TFA that includes a measure of gain, phase, coherence; (4) The first dCA assessment for ischemic stroke to be done within 24 hours of onset by TFA; (5) Clear reports of prognosis expressed as Modified Rankin Scale(mRS).

Information sources: Comprehensive literature searches of electronic bibliographic databases will be conducted in Pubmed, EMBASE, Web of Science, China National Knowledge Infrastructure database, and the Cochrane Library. No time restrictions will be placed on the date of publication.The following mesh terms were used: "cerebral autoregulation", "transfer function analysis", and "stroke". Furthermore, a manual search was carried out from all references cited in original studies and in all reviews identified. We will modify our search strategy to suit each database. Results from the database searches will be merged using an electronic reference manager to facilitate the removal of duplicates.

Main outcome(s): (1) TFA assessment: gain, phase, coherence; (2) prognosis assessment: $\mathrm{mRS}$ scores.
Quality assessment / Risk of bias analysis: We will use the mean differences as an effective indicator of measurement data and the confidence interval for each effect index will be set to $95 \%$. Publication bias will be assessed by funnel plots, Begg's and Egger's tests. Heterogeneity will be assessed with the aid of the use of searching at the qualities of contributors and medical characters in the blanketed foremost studies.Meanwhile, we will choose between fixed effects and random effects meta-analysis according to 12 statistics.A systematic review will be carried out instead of a meta-analysis when reviewers assess that the heterogeneity is considerable or lacks the quantitative data required for metaanalysis.

Strategy of data synthesis: We will describe and summarize the results of all included studies in a table and use REVMAN v5.4 software for the meta-analysis.

Subgroup analysis: When there is potential heterogeneity in this study, if all the information included in the study was available, we could perform subgroup analysis based on the sex, age, impaired vessels, impaired hemisphere and assessment time of the included subjects.

Sensitivity analysis: A sensitivity analysis will be performed to assess the robustness of the included results. If the results are unstable, studies with a high risk of bias will be excluded.

\section{Country(ies) involved: China.}

Keywords: meta-analysis, cerebral autoregulation, transfer function analysis, stroke, prognosis.

Contributions of each author:

Author 1 - Kai Wang.

Email: 550624487@qq.com

Author 2 - Zhiguo Lv.

Email: Ivzhuolunvip@163.com

Author 3 - Peng Xu.

Email: drxupeng@sina.com

Author 4 - Baitong Wang.

Email: 676314174@qq.com 
Author 5 - Aanqi Hou.

Email: 591855750@qq.com

Author 6 - Tianye Lan.

Email: lantianye-x@163.com

Author 7 - Dongmei Zhang.

Email: 1036878921@qq.com

Author 8 - Jian Wang.

Email: jian-w222@163.com 